\title{
LIE ALGEBROID GENERALIZATION OF GEOMETRIC MECHANICS
}

\author{
JOSÉ F. CARIÑENA \\ Departamento de Física Teórica, Universidad de Zaragoza \\ 50009, Zaragoza, Spain \\ E-mail:jfc@posta.unizar.es \\ EDUARDO MARTÍNEZ \\ Departamento de Matemática Aplicada, CPS, Universidad de Zaragoza \\ María de Luna 3, 50015 Zaragoza, Spain \\ E-mail: emf@posta.unizar.es
}

\begin{abstract}
Some recent progress in the geometric foundations of mechanics in Lie algebroids, and in particular in Lagrangian mechanics, is reported. We also develop the variational approach to classical mechanics in Lie algebroids.
\end{abstract}

1. Introduction. The concept of Lie algebroid is the infinitesimal version of that of Lie groupoid structure, which has recently been shown to play a relevant role in the quantization process [1]. It is in some sense a generalization of the concept of tangent bundle, on the one hand, and of Lie algebra on the other. Both of these last concepts have been playing an important role in the geometrical foundations of physics, a modern approach to physics whose interest has been growing almost continuously along the last years. Therefore, it suggests us to look for different fields or branches of physics where the concept of Lie algebroid may be relevant (see e.g. [2]-[5]). There are many applications in Quantum Field Theories, in particular in connection with Topological Field theories, mainly due to the relationship established in a paper by Vaintrob (see e.g. [6], [7]) among three different types of structures: Lie algebroid structures on a vector bundle $\tau: \mathcal{A} \rightarrow B$, homological vector fields on the supermanifold $\mathcal{M}=\left(B, \wedge^{\bullet} \mathcal{A}^{*}\right)$ and odd linear Poisson structures on $\mathcal{M}^{\prime}=\left(B, \wedge^{\bullet} \mathcal{A}\right)$. Information on such applications can be found in [8].

In this paper we will restrict ourselves to the applications in the geometric approach to classical mechanics. So, for the sake of completeness we will first present in Section 2

2000 Mathematics Subject Classification: Primary 81S10; Secondary 58H05.

Research partially supported by DGES project, PB96-0717.

The paper is in final form and no version of it will be published elsewhere. 
a quick review of the framework of classical mechanics, before proceeding in Section 3 to introduce the relevant definitions and properties of the Lie algebroid structures, and in Section 4 we analyze the exterior algebra of a Lie algebroid and a particularly important tool: the exterior differential associated in the space of sections of the dual of a Lie algebroid. In Section 5 we report the approach to classical mechanics in Lie algebroids proposed in [2] and [3]. After pointing out the deficiencies of such approach we explain in Section 6 the prolongation of a Lie algebroid that is convenient for the generalization in the case of Lie algebroids of the standard geometrical mechanics, which will be carried out following the same pattern as the standard approach. Finally, in the last section we analyze how to implement the variational approach to classical mechanics in Lie algebroids.

2. The general framework of classical mechanics. The dynamical evolution is described in Newtonian mechanics by a system of second order differential equations involving $n$ variables: $f^{i}=\frac{d^{2} x^{i}}{d t^{2}}$, where $i=1, \ldots, n$, which can be rewritten as a first order system in $2 n$ variables:

$$
\frac{d x^{i}}{d t}=v^{i}, \quad \frac{d v^{i}}{d t}=f^{i}
$$

In geometric terms this amounts to consider a manifold whose elements have positions and velocities as coordinates, and especial vector fields

$$
\Gamma=v^{i} \frac{\partial}{\partial x^{i}}+f^{i}(x, v) \frac{\partial}{\partial v^{i}} .
$$

On the other hand, the main assumption on which Lagrangian mechanics is based is a variational principle for the action functional:

$$
A(\gamma)=\int_{t_{1}}^{t_{2}} L(\gamma(t), \dot{\gamma}(t), t) d t
$$

for fixed end-points curves. This leads to the Euler-Lagrange equations:

$$
\frac{\partial L}{\partial q^{i}}=\frac{d}{d t}\left(\frac{\partial L}{\partial \dot{q}^{i}}\right),
$$

that when the Lagrangian is regular, i.e. the matrix with elements

$$
W_{i j}=\frac{\partial^{2} L}{\partial \dot{q}^{i} \partial \dot{q}^{j}},
$$

is regular, they provide a system of second order differential equations. On the other hand, Hamiltonian mechanics is usually introduced by means of the Legendre transformation, assumed to be invertible. Then the variables $\dot{q}$ are substituted for $p$ given by $p_{j}=\partial L / \partial \dot{q}^{j}$, with inverse $\dot{q}^{j}=\phi^{j}(q, p)$. The Hamilton function in this phase space is defined as $H(q, p)=\sum_{i=1}^{n} p_{i} \phi^{i}(q, p)-L(q, \phi(q, p))$. The equations corresponding to those of EulerLagrange are Hamilton equations, which provide the integral curves of the vector field in phase space

$$
X_{H}=\frac{\partial H}{\partial p_{j}} \frac{\partial}{\partial q^{j}}-\frac{\partial H}{\partial q^{j}} \frac{\partial}{\partial p_{j}} .
$$


We can also consider such equations for any function $H$ as a starting point. From the geometric viewpoint the important fact is that there is a Poisson bracket in phase space:

$$
\{F, G\}(q, p)=\sum_{i=1}^{n}\left(\frac{\partial F}{\partial q^{i}} \frac{\partial G}{\partial p_{i}}-\frac{\partial G}{\partial q^{i}} \frac{\partial F}{\partial p_{i}}\right) .
$$

It provides a Lie algebra structure: it is a bilinear, skew-symmetric product satisfying Jacobi identity,

$$
\left\{F_{1},\left\{F_{2}, F_{3}\right\}\right\}+\left\{F_{2},\left\{F_{3}, F_{1}\right\}\right\}+\left\{F_{3},\left\{F_{1}, F_{2}\right\}\right\}=0,
$$

plus the additional property:

$$
\left\{F, G_{1} G_{2}\right\}=G_{1}\left\{F, G_{2}\right\}+G_{2}\left\{F, G_{1}\right\},
$$

and the non-degeneracy condition

$$
\{F, G\}=0, \forall G \Longrightarrow F=\text { const. }
$$

These properties characterize (non-degenerate) Poisson algebras. Poisson brackets allow to describe dynamical evolution as follows:

$$
\frac{d F}{d t}=\{F, H\}
$$

So, a constant of the motion is a function $F$ such that $\{F, H\}=0$ and canonical transformations are characterized by $\{\bar{F}, \bar{G}\}=\{F, G\}$.

The geometric framework for the description of classical (and even quantum) systems is the theory of Hamiltonian dynamical systems [9], [10]. They are triplets $(M, \omega, H)$, where $M$ is a differentiable manifold, $\omega \in Z^{2}(M)$ is a regular closed 2-form in $M$, and $H \in C^{\infty}(M)$ is a function called Hamiltonian. The non-degeneracy of the symplectic form $\omega$ means that the $C^{\infty}(M)$-linear map $\hat{\omega}: \mathfrak{X}(M) \rightarrow \bigwedge^{1}(M)$ defined by contraction, i.e. $\hat{\omega}(X)=i(X) \omega$, is invertible. The dynamics is then given by the vector field $X_{H}$ such that $i\left(X_{H}\right) \omega=d H$.

We recall that an infinitesimal symmetry of a Hamiltonian dynamical systems $(M, \omega, H)$ is given by a Hamiltonian vector field $X \in \mathfrak{X}(M)$ (i.e., $i(X) \omega \in B^{1}(M)$ ) such that $X H=0$. The important point is that Noether's theorem establishes a one-to-one correspondence between infinitesimal symmetries and constants of motion, i.e. : $X H=0$ and $i(X) \omega=d f$ if and only if $f$ is a constant of motion.

If $Q$ is a differentiable manifold, the cotangent bundle $T^{*} Q$ is endowed with a canonical exact symplectic structure as follows: $\omega_{0}=-d \theta_{0}$ where $\theta_{0}$ is defined by:

$$
\text { If } \alpha \in T_{q} Q \quad \text { and } \quad U \in T_{\alpha}\left(T^{*} Q\right), \quad \theta_{0 \alpha}(U)=\alpha_{q}\left(\pi_{* \alpha}(U)\right) \text {. }
$$

In local coordinates, $\theta_{0}=p_{i} d q^{i}$ and $\omega_{0}=d q^{i} \wedge d p_{i}$.

The 2 -form $\omega$ of a symplectic manifold $(M, \omega)$ being non-degenerate, the map $\hat{\omega}$ : $T M \rightarrow T^{*} M$ is an isomorphism and every object in $T M$ has a corresponding one in $T^{*} M$. The object associated to the 2 -form $\omega$ is a skew-symmetric bivector field $\Lambda$ defined by $\Lambda(\alpha, \beta)=\hat{\omega}\left(\hat{\omega}^{-1}(\alpha), \hat{\omega}^{-1}(\beta)\right)$. Closedness of $\omega$ translates into $[\Lambda, \Lambda]_{S}=0$, where $[\cdot, \cdot]_{S}$ means Schouten bracket. The Poisson bracket of two functions is defined by $\{F, G\}=\Lambda(d F, d G)$. We find in this way a skew-symmetric bilinear map $\{\cdot, \cdot\}$ : $C^{\infty}(M) \times C^{\infty}(M) \rightarrow C^{\infty}(M)$ satisfying (4), (5) and the non-degeneracy condition (6). 
As a generalization, a Poisson bracket is a skew-symmetric bilinear map $\{\cdot, \cdot\}$ : $C^{\infty}(M) \times C^{\infty}(M) \rightarrow C^{\infty}(M)$ in a manifold $M$ satisfying (4) and (5), and when (6) holds the Poisson bracket is said to be non-degenerate. We can define a bivector field $\Lambda$ in $M$ by $\Lambda(d F, d G)=\{F, G\}$, and as a consequence of the assumption (4) such bivector field satisfies $[\Lambda, \Lambda]_{S}=0$.

Given $L \in C^{\infty}(T Q)$, if $q \in Q$ and $v, w \in T_{q} Q$, we define a map $\mathcal{F} L: T Q \rightarrow T^{*} Q$ by

$$
\mathcal{F} L(v)=\left.\frac{d}{d t} L(q, v+t w)\right|_{t=0} .
$$

In local coordinates, $\left(q^{i}, v^{i}\right) \mapsto\left(q^{i}, \partial L / \partial v^{i}\right)$. The map $\mathcal{F} L$ can be used to pull-back the canonical 2-form in $T^{*} Q$ and then we define $\theta_{L}=\mathcal{F} L^{*} \theta_{0}$ and $\omega_{L}=\mathcal{F} L^{*} \omega_{0}$. The Lagrangian $L$ is said to be regular if $\mathcal{F} L$ is a local diffeomorphism, or in an equivalent way, if $\omega_{L}$ is symplectic.

A tangent bundle $\tau_{Q}: T Q \rightarrow Q$ is characterized (see e.g. [11] and [12]) by the existence of: the Liouville vector field $\Delta \in \mathfrak{X}(T Q)$ generating dilations along the fibres and a $(1,1)$-tensor field $S$, called vertical endomorphism, given respectively by:

$$
(\Delta f)(q, v)=\frac{d}{d t} f\left(q, e^{t} v\right)_{\mid t=0},
$$

where $f \in C^{\infty}(T Q)$ and

$$
S_{(q, v)} U=\xi^{(q, v)}\left(\tau_{*(q, v)} U\right), \forall U \in T_{(q, v)}(T Q), v \in T_{q} Q,
$$

where $\xi^{(q, v)}: T_{q} Q \rightarrow T_{(q, v)}(T Q)$ denotes the vertical lift defined as follows:

$$
\xi^{(q, v)}(w) f=\frac{d}{d t} f(v+t w)_{\mid t=0} .
$$

In a natural coordinate system for $T Q$, induced from a chart in $Q$,

$$
\Delta=v^{i} \frac{\partial}{\partial v^{i}}, \quad S=\frac{\partial}{\partial v^{i}} \otimes d q^{i} .
$$

The image under $S$ of a section for $\tau_{T Q}: T(T Q) \rightarrow T Q$, a vector field in $T Q$, is a new vector field in $T Q$, again. This correspondence will also be denoted $S$. This tensor can be used to construct a 1 -form $d L \circ S$ which turns out to be the 1 -form $\theta_{L}$ associated to a Lagrangian $L \in C^{\infty}(T Q)$, i.e. $\theta_{L}=d L \circ S$. In this way the regular Lagrangians provide very interesting examples of $\operatorname{HDS},\left(T Q, \omega_{L}, E_{L}\right)$ with $E_{L}=\Delta L-L$. The dynamics is given by the vector field $X_{L}$ such that $i\left(X_{L}\right) \omega_{L}=d E_{L}$.

There is another vector bundle structure on $T(T Q)$ given by $T \tau_{Q}: T(T Q) \rightarrow T Q$. Vector fields on $T Q$ that are also sections for $T \tau_{Q}$ are called second order differential equations (SODE). They are characterized by $S(X)=\Delta$ and in tangent bundle coordinates look like in (2). Moreover, if $L$ is regular, $X_{L}$ is a SODE.

\section{Lie algebroids}

Definition 1. A Lie algebroid with base $B$ is a vector bundle $\mathcal{A}$ with base $B$ together with a Lie algebra structure in the space of its sections given by a Lie product $[\cdot, \cdot]_{\mathcal{A}}$ and a bundle map, called anchor, $\rho: \mathcal{A} \rightarrow T B$, inducing a map between the corresponding 
spaces of sections, to be denoted with the same name and symbol, such that

$$
\rho\left([X, Y]_{\mathcal{A}}\right)=[\rho(X), \rho(Y)], \quad[X, \varphi Y]_{\mathcal{A}}=\varphi[X, Y]_{\mathcal{A}}+(\rho(X) \varphi) Y,
$$

for any pair of sections of $\mathcal{A}, X, Y$, and each continuous function $\varphi$ defined in $B$.

When we consider adapted coordinates in $\mathcal{A},\left(x^{1}, \ldots, x^{n}, \lambda^{1}, \ldots, \lambda^{r}\right)$, where $\left(x^{1}, \ldots, x^{n}\right)$ are coordinates in the base manifold and $\left(\lambda^{1}, \ldots, \lambda^{r}\right)$ coordinates in the fibres associated with the choice of a basis of local sections of the Lie algebroid, $\left\{\xi_{\alpha} \mid \alpha=\right.$ $1, \ldots, r\}$, then

$$
\left[\xi_{\alpha}, \xi_{\beta}\right]_{\mathcal{A}}=\sum_{\gamma=1}^{r} c_{\alpha \beta}{ }^{\gamma} \xi_{\gamma}, \quad \alpha, \beta=1, \ldots, r,
$$

and the coordinate expression of the anchor map is

$$
\rho\left(\xi_{\alpha}\right)=\sum_{i=1}^{n} a^{i}{ }_{\alpha} \frac{\partial}{\partial x^{i}}, \quad \alpha=1, \ldots, r,
$$

with $c_{\alpha \beta}{ }^{\gamma}$ and $a_{\alpha}^{i}$ being the so called structure functions of the Lie algebroid.

The simplest example is that of a Lie algebra $\mathfrak{g}$ considered as a vector bundle over a single point.

Another simple example is the tangent bundle $T B$, when choosing the identity as anchor map $\rho$ and the commutator of vector fields as $[\cdot, \cdot]_{\mathcal{A}}$. With the usual coordinates $\left(q^{i}, v^{i}\right)$ in $T B$ induced from coordinates $\left(q^{i}\right)$ in the base $B$, the structure functions are $c_{i j}{ }^{k}=0, a_{i j}=\delta_{i j}$. However, in arbitrary coordinates in $T B$ the structure functions do not vanish. Moreover, an integrable subbundle of $T B$ with the inclusion as anchor map and the Lie product on the space of sections induced from that of $T B$ is also a simple example of Lie algebroid.

There exist alternative Lie algebroid structures on the tangent bundle $T B$. We recall that given a Lie algebra $\mathfrak{g}$ we can consider it as a $\mathfrak{g}$-module by means of the adjoint action. The linear mappings $A: \mathfrak{g} \rightarrow \mathfrak{g}$ are 1-cochains and the coboundary of such 1-cochain (see e.g. $[13,14,15])$ is

$$
(\delta A)\left(a_{1}, a_{2}\right)=\left[a_{1}, A\left(a_{2}\right)\right]-\left[a_{2}, A\left(a_{1}\right)\right]-A\left(\left[a_{1}, a_{2}\right]\right) .
$$

What is very relevant is that for any linear map $A, \delta A$ defines a skew-symmetric bilinear map, which can be denoted $[\cdot, \cdot]_{A}$ :

$$
\left[a_{1}, a_{2}\right]_{A}=\left[A\left(a_{1}\right), a_{2}\right]+\left[a_{1}, A\left(a_{2}\right)\right]-A\left(\left[a_{1}, a_{2}\right]\right) .
$$

The Nijenhuis torsion of $A$ is defined by

$$
T(A)\left(a_{1}, a_{2}\right)=A\left(\left[a_{1}, a_{2}\right]_{A}\right)-\left[A\left(a_{1}\right), A\left(a_{2}\right)\right],
$$

i.e.

$$
T(A)\left(a_{1}, a_{2}\right)=A\left(\left[a_{1}, A\left(a_{2}\right)\right]-\left[a_{2}, A\left(a_{1}\right)\right]-A\left(\left[a_{1}, a_{2}\right]\right)\right)-\left[A\left(a_{1}\right), A\left(a_{2}\right)\right],
$$

and $A$ is said to be a Nijenhuis tensor if its torsion vanishes: $T(A)=0$.

The main theorem (see [16]) is that if $A$ is a Nijenhuis tensor, then $\delta A$ defines a new Lie bracket which is compatible with the original Lie algebra structure, and $A$ is a Lie algebra homomorphism of $\left(\mathfrak{g},[\cdot, \cdot]_{A}\right)$ into $(\mathfrak{g},[\cdot, \cdot])$. 
We can now consider the case of $\mathfrak{g}$ being the Lie algebra of vector fields on a manifold $B$. A Nijenhuis structure on $B$ is a $(1,1)$-tensor field $N$ with vanishing Nijenhuis torsion, where the Nijenhuis torsion is defined by

$$
T(N)(X, Y)=-[N X, N Y]+N([N X, Y]+[X, N Y])-N^{2}[X, Y],
$$

where $X, Y \in \mathfrak{X}(M)$, so $(B, N)$ is a manifold with Nijenhuis structure if

$$
T(N)(X, Y)=0 \text {. }
$$

A Nijenhuis structure on a manifold $B$ defines an alternative Lie algebroid structure on $T B$ with bracket

$$
[X, Y]_{N}=[N X, Y]+[X, N Y]-N[X, Y]
$$

and anchor map given by $\rho=N: T B \rightarrow T B$, because, as indicated above, the bracket $[\cdot, \cdot]_{N}$ satisfies Jacobi identity ( $N$ is a assumed to be a Nijenhuis tensor) and for any function $f \in C^{\infty}(B)$,

$$
[X, f Y]_{N}=f[X, Y]_{N}+((N X) f) Y .
$$

As an interesting example, the vertical endomorphism in a tangent bundle is a Nijenhuis tensor in $T B$ and it may be used to endow the tangent bundle $\tau_{T B}: T(T B) \rightarrow T B$ with an alternative algebroid structure, for which

$$
[X, Y]_{S}=[S X, Y]+[X, S Y]-S[X, Y]
$$

Another very interesting example of Lie algebroid is that of the cotangent bundle of a Poisson manifold $(P, \Lambda)$. It is a Lie algebroid when we define [17]

$$
\{\alpha, \beta\}=\mathcal{L}_{\hat{\Lambda}(\alpha)} \beta-\mathcal{L}_{\hat{\Lambda}(\beta)} \alpha-d[\Lambda(\alpha, \beta)],
$$

and the anchor $\rho$ is given by $\rho(\alpha) f=\Lambda(\alpha, d f)$. Here $\hat{\Lambda}(\alpha)$ is defined by $\langle\beta, \hat{\Lambda}(\alpha)\rangle=$ $\Lambda(\alpha, \beta)$. This Lie bracket in the space of 1 -forms satisfies $\{d f, d g\}=d\{f, g\}$, for any pair of functions in $P$. Notice that a function $H \in C^{\infty}(P)$ defines a dynamical vector field $X_{H}=\{\cdot, H\}$ and that if two 1-forms are invariant under $X_{H}$, then $\{\alpha, \beta\}$ is also invariant. In other words, the Lie bracket so defined in the space of 1-forms in a Poisson manifold, furnishes a method for associating an invariant 1-form with a couple of invariant 1-forms much in the same way as the commutator of two symmetry vector fields for $X_{H}$ is again a symmetry.

A remarkable property is that if $\mathcal{A} \rightarrow B$ is a vector bundle with a structure of Lie algebroid, then the dual bundle $\mathcal{A}^{*} \rightarrow B$ is endowed with a homogeneous (of degree minus one) Poisson structure $\Lambda, \mathcal{L}_{Z} \Lambda=-\Lambda$, with $Z$ being the vector field generating dilations along the fibres of $\mathcal{A}^{*}$, and the converse property is also true:

Theorem 1. The vector bundle $\pi: \mathcal{A} \rightarrow B$ is an algebroid with base $B$ and anchor $\rho: \mathcal{A} \rightarrow T B$ if and only if the dual bundle $\mathcal{A}^{*} \rightarrow B$ is a Poisson manifold whose linear functions form a Lie subalgebra.

In particular, if $B$ reduces to a point and $\mathcal{A}=\mathfrak{g}$ is a Lie algebra, we find the well-known case of the canonical Poisson structure on the dual of the Lie algebra, $\mathfrak{g}^{*}$. As another example, when $\mathcal{A}=T B$, then $\mathcal{A}^{*}=T^{*} B$ and the Poisson structure in $T^{*} B$ is the canonical symplectic structure on the cotangent bundle. 
In order to describe the Poisson structure of $\mathcal{A}^{*}$ it suffices to give the Poisson brackets of a class of functions such that their differentials span the cotangent space at each point of $\mathcal{A}^{*}$. Such a class of functions is given by functions which are affine in the fibres. Those functions which are constant on the fibres, basic functions, correspond to pull-back of functions on $B$. On the other hand, functions that are linear in the fibres can be identified with sections of $\mathcal{A}$. If $f$ and $g$ are functions on $B$ and $\xi$ and $\eta$ are sections in $\mathcal{A}$, their bracket relations as functions on $\mathcal{A}^{*}$ are

$$
\{f, g\}=0, \quad\{f, \xi\}=\rho(\xi) f, \quad\{\xi, \eta\}=[\xi, \eta] .
$$

Given adapted coordinates in $\mathcal{A},\left(q^{1}, \ldots, q^{n}, \lambda^{1}, \ldots, \lambda^{r}\right)$, and with the corresponding dual coordinates, $\left(q^{1}, \ldots, q^{n}, \mu_{1}, \ldots, \mu_{r}\right)$, the defining relations of the Poisson structure are

$$
\left\{q^{i}, q^{j}\right\}=0, \quad\left\{\mu_{\alpha}, \mu_{\beta}\right\}=\sum_{\gamma \in \Lambda} c_{\alpha \beta}{ }^{\gamma} \mu_{\gamma}, \quad\left\{q^{i}, \mu_{\alpha}\right\}=a^{i}{ }_{\alpha}
$$

4. Exterior algebra of a Lie algebroid. Let $\left(\mathcal{A}, \rho,[\cdot, \cdot]_{\mathcal{A}}\right)$ be a Lie algebroid, and consider the exterior algebra of such a Lie algebroid via the exterior algebra $\bigwedge^{\bullet} \mathcal{A}^{*}$ of the dual of $\mathcal{A}$. The sections of $\bigwedge^{\bullet} \mathcal{A}^{*}$ are called $\mathcal{A}$-forms. Their set is a $C^{\infty}(B)$-module, $\left(\Gamma\left(\bigwedge^{\bullet} \mathcal{A}^{*}\right)\right)$. An $\mathcal{A}$ - $(k)$-form is a form such that $\theta \in \Gamma\left(\bigwedge^{k} \mathcal{A}^{*}\right)$.

We can define an " $\mathcal{A}$-exterior" differential operator which takes an $\mathcal{A}$ - $(k)$-form into an $\mathcal{A}$ - $(k+1)$-form as follows:

If $f \in C^{\infty}(B)$, then $\langle d f, v\rangle=\rho(v) f$, while if $\theta$ is a $\mathcal{A}$-(k)-form, $k \geq 1, d_{\mathcal{A}}$ is given by

$$
\begin{aligned}
d_{\mathcal{A}} \theta\left(v_{1}, \ldots, v_{k+1}\right) & =\sum_{i}(-1)^{i+1} \rho\left(v_{i}\right) \theta\left(v_{1}, \ldots, \hat{v}_{i}, \ldots, v_{k+1}\right)+ \\
& +\sum_{i<j}(-1)^{i+j} \theta\left(\left[v_{i}, v_{j}\right]_{\mathcal{A}}, v_{1}, \ldots, \hat{v}_{i}, \ldots, \hat{v}_{j}, \ldots v_{k+1}\right),
\end{aligned}
$$

for $v_{1}, \ldots, v_{k+1} \in \Gamma(\mathcal{A})$.

The Lie algebroid axioms imply that $d_{\mathcal{A}}$ is a $C^{\infty}(B)$-multilinear map such that $d_{\mathcal{A}}^{2}=0$ and is a super-derivation of degree 1 , i.e.

$$
d_{\mathcal{A}}(\theta \wedge \omega)=d_{\mathcal{A}} \theta \wedge \omega+(-1)^{|\theta|} \theta \wedge d_{\mathcal{A}} \omega .
$$

Observe that an exterior derivation $d_{\mathcal{A}}$ satisfying $d_{\mathcal{A}}^{2}=0$ on $\Gamma\left(\Lambda^{\bullet} \mathcal{A}^{*}\right)$ is equivalent to the Lie algebroid structure on $\mathcal{A}$; furthermore, one can see $\Gamma\left(\Lambda^{\bullet} \mathcal{A}^{*}\right)$ as the algebra of functions on the superspace $\Pi \mathcal{A}$ (where $\Pi$ denotes parity reversion), so the differential $d_{\mathcal{A}}$ can be interpreted as an odd vector field of degree 1.

In the example of $\mathcal{A}=\mathfrak{g}$, where $\rho=0$ and $\left[\xi_{\alpha}, \xi_{\beta}\right]_{\mathcal{A}}=c_{\alpha \beta}{ }^{\gamma} \xi_{\gamma}$, then the differential operator is defined by:

$$
d_{\mathcal{A}} \theta\left(v_{1}, \ldots, v_{k+1}\right)=\sum_{i<j}(-1)^{i+j} \theta\left(\left[v_{i}, v_{j}\right]_{\mathcal{A}}, v_{1}, \ldots, \hat{v}_{i}, \ldots, \hat{v}_{j}, \ldots, v_{k+1}\right),
$$

where $v_{i} \in \mathfrak{g}$, i.e., this is the Chevalley operator (Chevalley-Eilenberg), and generate the Lie algebra cohomology of $\mathfrak{g}$.

When we choose $\mathcal{A}=T B$, the anchor is the identity and the commutator of vector fields the product $[\cdot, \cdot]_{\mathcal{A}}$, the exterior operator reduces to 


$$
\begin{gathered}
d_{\mathcal{A}} \theta\left(X_{1}, \ldots, X_{i}, \ldots, X_{k+1}\right)=\sum_{i}(-1)^{i+1} X_{i} \theta\left(X_{1}, \ldots, \hat{X}_{i}, \ldots, X_{k+1}\right) \\
+\sum_{i<j}(-1)^{i+j} \theta\left(\left[X_{i}, X_{j}\right]_{\mathcal{A}}, X_{1}, \ldots, \hat{X}_{i}, \ldots, \hat{X}_{j}, \ldots X_{k+1}\right),
\end{gathered}
$$

where $X_{i} \in \mathfrak{X}(B)$, which is the de Rham operator, and its associated cohomology is de Rham cohomology.

We can also consider the Lie algebroid structure defined in $\tau_{T B}: T(T B) \rightarrow T B$ by the vertical endomorphism $S$, and then

$$
\begin{aligned}
& d_{S} \theta\left(X_{1}, \ldots, X_{i}, \ldots, X_{k+1}\right)=\sum_{i}(-1)^{i+1} S\left(X_{i}\right) \theta\left(X_{1}, \ldots, \hat{X}_{i}, \ldots, X_{k+1}\right) \\
& \quad+\sum_{i<j}(-1)^{i+j} \theta\left(\left[X_{i}, X_{j}\right]_{S}, X_{1}, \ldots, \hat{X}_{i}, \ldots, \hat{X}_{j}, \ldots X_{k+1}\right),
\end{aligned}
$$

which is nothing but the expression (2.4.23) for $d_{S}$ used in [18], but remark that it appears here in a natural way as the exterior differential operator associated to this alternative algebroid structure.

5. Applications in geometrical mechanics. The geometric approach to classical mechanics considers that the configuration space is a differentiable manifold $Q$ and the tangent bundle $\tau_{Q}: T Q \rightarrow Q$ is the velocity-phase space.

The vector bundle $\tau_{T Q}: T(T Q) \rightarrow T Q$ is endowed with another vector bundle structure given by $T \tau_{Q}: T(T Q) \rightarrow T Q$. Vector fields on $T Q$ that are also sections for $T \tau_{Q}$ are called second order differential equations (SODE). The set of such SODE vector fields will be denoted $\mathfrak{X}_{s}(T Q)$. Note that $X \in \mathfrak{X}_{s}(T Q)$ if and only if $S(X)=\Delta$.

As indicated before, we can see a Lie algebroid over $B$ as a new "tangent bundle" for $B$. As $T Q$ plays a relevant role in the geometrical approach to the Lagrangian formalism of classical mechanics, we can try to extend the formalism to this new framework of Lie algebroids. Now $B$ will play the role of configuration space $Q$ and $\mathcal{A}$ will take the place of velocity phase space $T Q$.

The first method of generalizing the theory is the one proposed in $[2,3]$ and is based on the generalized Legendre transformation. So, if $L$ is a real-valued function on the Lie algebroid $\mathcal{A}$ over $M$, the Legendre mapping $\mathcal{F} L: \mathcal{A} \rightarrow \mathcal{A}^{*}$ of $L$ is defined as the fibre derivative of $L$, i.e., if $b \in B$ and $L_{b}: \mathcal{A} \rightarrow \mathbb{R}$ is given by the restriction to $\pi^{-1}(b)$,

$$
\mathcal{F} L(v)(w)=L_{b * v}(w)=\left.\frac{d}{d t} L_{b}(v+t w)\right|_{t=0} .
$$

The action $A$ is defined by $A(v)=\langle\mathcal{F} L(v), v\rangle$, and the energy $E$ is given by $A-L$.

When $\mathcal{F} L$ is a local diffeomorphism we will say that $L$ is a regular Lagrangian. In this case $\mathcal{F} L$ can be used to pull back the Poisson structure from $\mathcal{A}^{*}$ to a Poisson structure on $\mathcal{A}$ which we will call the Lagrange Poisson structure. $\{\cdot, E\}$, is called the Lagrangian vector field associated with the regular Lagrangian $L$.

In standard coordinates, if $L(q, \lambda)$, then $\mathcal{F} L$ is defined by $\mu_{\alpha}=\partial L / \partial \lambda^{\alpha}$, so that

$$
\left\{q^{i}, q^{j}\right\}=0, \quad\left\{\frac{\partial L}{\partial \lambda^{\alpha}}, \frac{\partial L}{\partial \lambda^{\beta}}\right\}=\sum_{\gamma=1}^{r} c_{\alpha \beta} \gamma \frac{\partial L}{\partial \lambda^{\gamma}}, \quad \text { and } \quad\left\{q^{i}, \frac{\partial L}{\partial \lambda^{\beta}}\right\}=a_{\beta}^{i} .
$$


The action and the energy functions are, respectively,

$$
A=\sum_{\alpha=1}^{r} \lambda^{\alpha} \frac{\partial L}{\partial \lambda^{\alpha}} \quad \text { and } \quad E=\sum_{\alpha=1}^{r} \lambda^{\alpha} \frac{\partial L}{\partial \lambda^{\alpha}}-L
$$

Lagrange's equations in Poisson bracket form are

$$
\frac{d q^{i}}{d t}=\left\{q^{i}, E\right\} \quad \text { and } \quad \frac{d \lambda^{\alpha}}{d t}=\left\{\lambda^{\alpha}, E\right\} .
$$

Using the bracket relation

$$
\left\{q^{i}, L\right\}=\sum_{\beta=1}^{r}\left\{q^{i}, \lambda^{\beta}\right\} \frac{\partial L}{\partial \lambda^{\beta}},
$$

we can rewrite the first Lagrange equation as follows:

$$
\begin{aligned}
\frac{d q^{i}}{d t} & =\left\{q^{i}, E\right\}=\left\{q^{i}, \sum_{\beta=1}^{r} \lambda^{\beta} \frac{\partial L}{\partial \lambda^{\beta}}-L\right\} \\
& =\sum_{\beta=1}^{r}\left\{q^{i}, \frac{\partial L}{\partial \lambda^{\beta}}\right\} \lambda^{\beta}+\sum_{\beta=1}^{r}\left\{q^{i}, \lambda^{\beta}\right\} \frac{\partial L}{\partial \lambda^{\beta}}-\left\{q^{i}, L\right\} \\
& =\sum_{\beta=1}^{r}\left\{q^{i}, \frac{\partial L}{\partial \lambda^{\beta}}\right\} \lambda^{\beta}=\sum_{\beta=1}^{r} a_{\beta}^{i} \lambda^{\beta} \\
& =\sum_{\beta=1}^{r}\left(\rho\left(\xi_{\beta}\right) \cdot q^{i}\right) \lambda^{\beta}=\rho\left(\sum_{\beta=1}^{r} \lambda^{\beta} \xi_{\beta}\right) \cdot q^{i} .
\end{aligned}
$$

A tangent vector $v$ to a Lie algebroid $\mathcal{A}$ at a point $\lambda$ is called admissible when $T \operatorname{pr}_{\mathcal{A}}(v)=\rho(v)$, where $T \operatorname{pr}_{\mathcal{A}}: T \mathcal{A} \rightarrow T M$ is the derivative of the vector bundle projection $\operatorname{pr}_{\mathcal{A}}: \mathcal{A} \rightarrow M$. A curve in $\mathcal{A}$ is called admissible if its tangent vectors are admissible. A vector field $X$ on $\mathcal{A}$ is a second order differential equation (SODE) if its values are all admissible vectors. Thus, a vector field on $\mathcal{A}$ is a second order differential equation iff all its integral curves are admissible. The equation $\frac{d q^{i}}{d t}=\rho\left(\sum_{\beta \in \Lambda} \lambda^{\beta} \xi_{\beta}\right) \cdot q^{i}$ shows that the Lagrangian vector field associated with any regular Lagrangian is a second order differential equation in the preceding sense.

The main difficulty of this approach is that it is only possible for regular Lagrangians, otherwise $\mathcal{F} L$ is not invertible and the pull-back of the Poisson structure in $\mathcal{A}^{*}$ is not well defined. Moreover, it is quite difficult the analysis of questions such as the study of equivalence of Lagrangians. So we will present next the alternative approach developed in [4], still valid for singular Lagrangians and allowing us a new perspective.

6. The prolongation of a Lie algebroid. In the generalization of the Lagrangian formalism, when the Lie algebroid $\mathcal{A}$ is playing the role of $T Q$ there is no possibility of defining an object corresponding to the vertical endomorphism: the dimension of the fibres does not coincide with the dimension of the tangent space at a point in the base. The tangent bundle $T \mathcal{A}$ will be a $2(n+m)$-dimensional space with coordinates $\left(x^{i}, y^{\alpha}, \dot{x}^{i}, \dot{y}^{\alpha}\right)$ and the two sets of coordinates $\dot{x}^{i}$ and $y^{\alpha}$ cannot be interchanged. 
We need to look for a new bundle $\mathcal{L} \mathcal{A}$ playing a role similar to the one played by $T(T Q)$. Let us consider the total space of the induced bundle $\rho^{*}(T \tau)$, namely

$$
\mathcal{L} \mathcal{A}=\{(b, v) \in \mathcal{A} \times T \mathcal{A} \mid \rho(b)=T \tau(v)\} .
$$

and consider it as a vector bundle over $\mathcal{A}$ w.r.t. $\tau_{1}(b, v)=\tau(v)=a$.

We will use the redundant notation $(a, b, v)$ to denote the element $(b, v) \in \mathcal{L} \mathcal{A}$ such that $v \in T_{a} \mathcal{A}$. Then, $\tau_{1}(a, b, v)=a$ and

$$
\mathcal{L} \mathcal{A}=\left\{(a, b, v) \mid v \in T_{a} \mathcal{A}, \tau(a)=\tau(b), \rho(b)=T \tau(v)\right\} .
$$

We recall that $\rho^{*}(T \tau)$ is defined by the commutative diagram

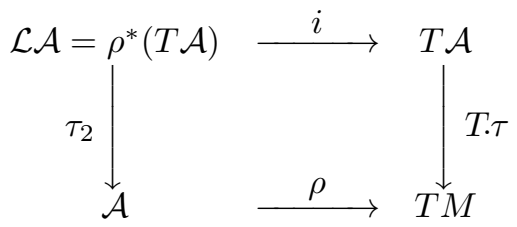

The remarkable point is that $\mathcal{L} \mathcal{A}$ can be considered as a vector bundle over $\mathcal{A}$ in two inequivalent ways, either by means of the projection $\tau_{1}: \mathcal{L} \mathcal{A} \rightarrow \mathcal{A}$ given by $\tau_{1}(a, b, v)=a$ or by the projection $\tau_{2}: \mathcal{L} \mathcal{A} \rightarrow \mathcal{A}$ is given by $\tau_{2}(a, b, v)=b$. The projection $\tau_{2}: \mathcal{L} \mathcal{A} \rightarrow \mathcal{A}$ plays the role of $T \tau_{Q}: T(T Q) \rightarrow T Q$.

Even more, we can define the projection $\rho^{1}: \mathcal{L} \mathcal{A} \rightarrow T \mathcal{A}$ on the third argument, $\rho^{1}(a, b, v)=v$. It will be the anchor of the prolonged algebroid.

Vertical elements of $\mathcal{L} \mathcal{A}$ are those of $\operatorname{ker} \tau_{2}:(a, 0, v) \in \mathcal{L} \mathcal{A}$ such that $v \in \operatorname{ker} \tau_{* a}$. The set of vertical elements of $\mathcal{L} \mathcal{A}$ is the subbundle denoted $\operatorname{Ver}(\mathcal{L} \mathcal{A})$.

If $z$ is vertical, then $\rho^{1}(z)$ is vertical, but the converse is not true, but only when $\rho$ is injective

In the coordinates we mentioned before, if the coordinates of $a$ are $\left(x^{i}, y^{\alpha}\right)$, those of $b$ are $\left(x^{i}, z^{\alpha}\right)$, and $v$ is given by

$$
v=\bar{v}^{i} \frac{\partial}{\partial \dot{x}^{i}}+v^{\alpha} \frac{\partial}{\partial z^{\alpha}}
$$

then the condition $\rho(b)=T \tau(v)$ is written

$$
\bar{v}^{i}=\rho_{\alpha}^{i} z^{\alpha},
$$

in such a way that the coordinates of $(a, b, v)$ are $\left(x^{i}, y^{\alpha}, z^{\alpha}, v^{\alpha}\right)$.

As $\mathcal{A}$ is a vector bundle the fibres can be identified with the vertical tangent spaces via the vertical lift

$$
b_{a}^{V} F=\frac{d}{d t} F(a+t b)_{\mid t=0} .
$$

We can define the vertical lift $\xi^{V}: \mathcal{A} \times_{M} \mathcal{A} \rightarrow \mathcal{L} \mathcal{A}$ by

$$
\xi^{V}(a, b)=\left(a, 0, b_{a}^{V}\right) .
$$

This is a vector bundle isomorphism from $\operatorname{pr}_{1}: \mathcal{A} \times_{M} \mathcal{A} \rightarrow \mathcal{A}$ to $\tau_{1}: \operatorname{Ver}(\mathcal{L} \mathcal{A}) \rightarrow \mathcal{A}$. Each $\sigma \in \operatorname{Sec}(\tau)$ defines a section $\sigma^{V} \in \operatorname{Sec}\left(\tau_{1}\right)$, called vertical lift of $\sigma$, by

$$
\sigma^{V}(a)=\xi^{V}(a, \sigma(\tau(a))) .
$$


A section $\eta$ of $\mathcal{L} \mathcal{A}$ is said to be projectable if there exists a section $\sigma$ for $\tau$ such that $\tau_{2} \circ \eta=\sigma \circ \tau$. Then the vector field $\rho^{1}(\eta)$ is projectable to $M$.

Let us remark that $z=(a, b, v) \in \mathcal{L} \mathcal{A}$ is not determined by giving the action of $v$ on functions. Instead, $z$ can be given by the element $(a, b)$ of $\mathcal{A} \times{ }_{M} \mathcal{A}$ and the action of $v$ on linear functions.

If $\sigma \in \operatorname{Sec}(\tau)$, the complete lift $\sigma^{c} \in \operatorname{Sec}\left(\tau_{1}\right)$ is the section projecting onto $\sigma$ and satisfying

$$
\rho^{1}\left(\sigma^{c}\right)(\hat{\theta})=\widehat{d_{\sigma} \theta}
$$

Here $d_{\sigma}$ means

$$
d_{\sigma}=i_{\sigma} \circ d+d \circ i_{\sigma} .
$$

Coordinate expressions: If $a=\left(m^{i}, a^{\alpha}\right), b=\left(m^{i}, b^{\alpha}\right)$, then

$$
v=\left.\rho_{\alpha}^{i} b^{\alpha} \frac{\partial}{\partial x^{i}}\right|_{a}+\left.v^{\alpha} \frac{\partial}{\partial y^{\alpha}}\right|_{a},
$$

and denote the coordinates of $(a, b, v)$ by $\left(m^{i}, a^{\alpha}, b^{\alpha}, v^{\alpha}\right)$,

$$
\rho^{1}(x, y, z, v)=\left.\rho_{\alpha}^{i} z^{\alpha} \frac{\partial}{\partial x^{i}}\right|_{(x, y)}+\left.v^{\alpha} \frac{\partial}{\partial y^{\alpha}}\right|_{(x, y)} .
$$

A local basis of sections of $\mathcal{L} \mathcal{A}$ is given by:

$$
\begin{aligned}
\mathcal{X}_{\alpha}(a) & =\left(a, e_{\alpha}(\tau(a)),\left.\rho_{\alpha}^{i} \frac{\partial}{\partial x^{i}}\right|_{a}\right), \\
\mathcal{V}_{\alpha}(a) & =\left(a, 0,\left.\frac{\partial}{\partial y^{\alpha}}\right|_{a}\right) .
\end{aligned}
$$

If $V$ is the section of $\mathcal{L} \mathcal{A}, V(x, y)=\left(x^{i}, y^{\alpha}, Z^{\alpha}(x, y), V^{\alpha}(x, y)\right)$,

$$
\begin{aligned}
V & =Z^{\alpha} \mathcal{X}_{\alpha}+V^{\alpha} \mathcal{V}_{\alpha}, \\
\rho^{1}(V) & =\left.\rho_{\alpha}^{i} Z^{\alpha}(x, y) \frac{\partial}{\partial x^{i}}\right|_{(x, y)}+\left.V^{\alpha}(x, y) \frac{\partial}{\partial y^{\alpha}}\right|_{(x, y)} .
\end{aligned}
$$

The vertical and complete lifts of $\sigma=\sigma^{\alpha} e_{\alpha}$ are:

$$
\begin{aligned}
\sigma^{V} & =\sigma^{\alpha} \mathcal{V}_{\alpha}, \quad \text { and } \rho^{1}\left(\sigma^{V}\right)=\sigma^{\alpha} \frac{\partial}{\partial y^{\alpha}} . \\
\sigma^{c} & =\sigma^{\alpha} \mathcal{X}_{\alpha}+\left(\dot{\sigma}^{\alpha}-C_{\beta \gamma}^{\alpha} \sigma^{\beta} y^{\gamma}\right) \mathcal{V}_{\alpha}, \\
\rho^{1}\left(\sigma^{c}\right) & =\rho^{i}{ }_{\alpha} \sigma^{\alpha} \frac{\partial}{\partial x^{i}}+\left(\dot{\sigma}^{\alpha}-C_{\beta \gamma}^{\alpha} \sigma^{\beta} y^{\gamma}\right) \frac{\partial}{\partial y^{\alpha}} .
\end{aligned}
$$

THEOREM 2. There exists a Lie algebroid structure on $\tau_{1}: \mathcal{L} \mathcal{A} \rightarrow \mathcal{A}$ such that the anchor is $\rho^{1}$ and the bracket satisfies

$$
\left[\sigma^{V}, \eta^{V}\right]=0, \quad\left[\sigma^{V}, \eta^{c}\right]=[\sigma, \eta]^{V}, \quad\left[\sigma^{c}, \eta^{c}\right]=[\sigma, \eta]^{c},
$$

for any couple of sections $\sigma, \eta \in \operatorname{Sec}(\mathcal{A})$.

The structure functions of $\mathcal{L} \mathcal{A}$ are given by

$$
\begin{aligned}
\rho^{1}\left(\mathcal{X}_{\alpha}\right) & =\rho^{i}{ }_{\alpha} \frac{\partial}{\partial x^{i}}, \quad \rho^{1}\left(\mathcal{V}_{\alpha}\right)=\frac{\partial}{\partial y^{\alpha}} \\
{\left[\mathcal{X}_{\alpha}, \mathcal{X}_{\beta}\right] } & =c_{\alpha \beta}^{\gamma} \mathcal{X}_{\gamma}, \quad\left[\mathcal{X}_{\alpha}, \mathcal{V}_{\beta}\right]=0, \quad\left[\mathcal{V}_{\alpha}, \mathcal{V}_{\beta}\right]=0
\end{aligned}
$$


If $\left\{\mathcal{X}^{\alpha}, \mathcal{V}^{\alpha}\right\}$ denotes the dual basis, then

$$
d F=\rho_{\alpha}^{i} \frac{\partial F}{\partial x^{i}} \mathcal{X}^{\alpha}+\frac{\partial F}{\partial y^{\alpha}} \mathcal{V}^{\alpha}
$$

and in particular,

$$
d x^{i}=\rho_{\alpha}^{i} \mathcal{X}^{\alpha}, \quad d y^{\alpha}=\mathcal{V}^{\alpha},
$$

and the differential of sections of $(\mathcal{L} \mathcal{A})^{*}$ is determined by

$$
d \mathcal{X}^{\alpha}=-\frac{1}{2} c_{\beta \gamma}^{\alpha} \mathcal{X}^{\beta} \wedge \mathcal{X}^{\gamma}, \quad d \mathcal{V}^{\alpha}=0 .
$$

Related constructions to those given here has been considered in [19].

Liouville section and vertical endomorphism. The Liouville section $\Delta$ is the section of $\tau_{1}$ given by

$$
\Delta(a)=\xi^{V}(a, a)=\left(a, 0, a_{a}^{V}\right)
$$

The vertical endomorphism is an endomorphism of $\tau_{1}: \mathcal{L} \mathcal{A} \rightarrow \mathcal{A}$ defined by

$$
S(a, b, v)=\left(a, 0, b_{a}^{V}\right),
$$

and therefore

$$
S\left(\sigma^{V}\right)=0, \quad \text { and } \quad S\left(\sigma^{c}\right)=\sigma^{V}, \forall \sigma \in \operatorname{Sec}(\mathcal{A}) .
$$

As two important properties, $\operatorname{Im} S=\operatorname{ker} S=\operatorname{Ver}(\mathcal{L} \mathcal{A})$ and then $S^{2}=0$, and the Nijenhuis tensor of $S$ vanishes $N_{S}=0$. The coordinate expressions of $\Delta, \rho^{1}(\Delta)$ and $S$ are

$$
\Delta=y^{\alpha} \mathcal{V}_{\alpha}, \quad \rho^{1}(\Delta)=y^{\alpha} \frac{\partial}{\partial y^{\alpha}}, \quad S=\mathcal{V}_{\alpha} \otimes \mathcal{X}^{\alpha}
$$

A tangent vector $v \in T_{a} \mathcal{A}$ is called admissible if $T \tau_{a}(v)=\rho(a)$. A curve in $\mathcal{A}$ is admissible if its tangent vectors are admissible. Then $v \in T_{a} \mathcal{A}$ is admissible if and only if $(a, a, v) \in \mathcal{L} \mathcal{A}$, i.e.,

$$
\operatorname{Adm}=\left\{z \in \mathcal{L} \mathcal{A} \mid \tau_{1}(z)=\tau_{2}(z)\right\}
$$

The important result is that $\Gamma \in \operatorname{Sec}(\mathcal{L} \mathcal{A})$ takes admissible values iff $\tau_{2} \circ \Gamma=\mathrm{id} \mathcal{A}_{\mathcal{A}}$, or equivalently, iff $S(\Gamma)=\Delta$. Such a section is said to be a SODE on $\mathcal{A}$. In local coordinates,

$$
\begin{aligned}
\Gamma(x, y) & =y^{\alpha} \mathcal{X}_{\alpha}+f^{\alpha}(x, y) \mathcal{V}_{\alpha}, \\
\rho^{1}(\Gamma)(x, y) & =\left.\rho^{i}{ }_{\alpha} y^{\alpha} \frac{\partial}{\partial x^{i}}\right|_{(x, y)}+\left.f^{\alpha}(x, y) \frac{\partial}{\partial y^{\alpha}}\right|_{(x, y)} .
\end{aligned}
$$

The integral curves of the SODE $\Gamma$, i.e., the integral curves of $\rho^{1}(\Gamma)$, are to be determined by the system of differential equations

$$
\frac{d x^{i}}{d t}=\rho_{\alpha}^{i}(x) y^{\alpha}, \quad \frac{d y^{\alpha}}{d t}=f^{\alpha}(x, y) .
$$

Using the object $S$ and $\Delta$ so defined we can define a Lagrangian formalism in Lie algebroids as follows. Let $\theta_{L}$ be the section of $(\mathcal{L} \mathcal{A})^{*}$ given by $\theta_{L}=S(d L)$ which in coordinates looks $\theta_{L}=\frac{\partial L}{\partial y^{\alpha}} \mathcal{X}^{\alpha}$. The Cartan 2-form is defined by $\omega_{L}=-d \theta_{L}$, with coordinate expression

$$
\omega_{L}=\frac{\partial^{2} L}{\partial y^{\alpha} \partial y^{\beta}} \mathcal{X}^{\alpha} \wedge \mathcal{X}^{\beta}+\frac{1}{2}\left(\frac{\partial^{2} L}{\partial x^{i} \partial y^{\alpha}} \rho_{\beta}^{i}-\frac{\partial^{2} L}{\partial x^{i} \partial y^{\beta}} \rho_{\alpha}^{i}+\frac{\partial L}{\partial y^{\gamma}} C_{\alpha \beta}^{\gamma}\right) \mathcal{X}^{\alpha} \wedge \mathcal{V}^{\beta}
$$


The energy function $E_{L}$ and the dynamical equation are given, respectively by

$$
\begin{aligned}
E_{L} & =d_{\Delta} L-L=\frac{\partial L}{\partial y^{\alpha}} y^{\alpha}-L, \\
i_{\Gamma} \omega_{L} & =d E_{L} .
\end{aligned}
$$

For regular Lagrangians this equation can be rewritten as:

$$
d_{\Gamma}\left(\frac{\partial L}{\partial y^{\alpha}}\right)=\rho_{\alpha}^{i} \frac{\partial L}{\partial x^{i}}-c_{\alpha \beta}^{\gamma} y^{\beta} \frac{\partial L}{\partial y^{\gamma}}, \quad \alpha=1, \ldots, r,
$$

and it is easy to prove that $\Gamma$ is a second order differential equation field on the Lie algebroid $\mathcal{A}$. In this case, the dynamical equations can be rewritten as

$$
d_{\Gamma} \theta_{L}-d L=0
$$

7. Variational principle. The Lie algebra structure in the prolongation of a Lie algebroid has been defined in terms of brackets of complete and vertical lifts. While there are other ways to define that structure, one reason for doing so is to follow as close as possible the results in the case of the canonical Lie algebroid. In that case, the relations between complete and vertical lifts are fundamental in the development of the variational calculus, which after all is a basic ingredient of Lagrangian mechanics. In this section we will consider the variational problem and we will show that the variational equations defined by a regular Lagrangian $L$ are exactly the equations given in the last section.

Let $m_{0}$ and $m_{1}$ be two points of the base manifold $B$. We consider the set of admissible curves $\eta:\left[t_{0}, t_{1}\right] \rightarrow \mathcal{A}$ such that $\gamma\left(t_{0}\right)=m_{0}$ and $\gamma\left(t_{1}\right)=m_{1}$ for some fixed real numbers $t_{0}$ and $t_{1}$, and where $\gamma=\pi \circ \eta$. Given a Lagrangian $L \in C^{\infty}(\mathcal{A})$ we look for the critical points of the functional

$$
J(\eta)=\int_{a}^{b} L(\eta(t)) d t
$$

on that set. This is a constrained problem, since the curves we consider are restricted to be admissible, i.e. they satisfy the constraints

$$
\dot{x}^{i}=\rho_{\alpha}^{i} y^{\alpha} .
$$

A first attempt to solve our variational problem is to use Lagrange multipliers method, as it is usually done in physics. Nevertheless, the method fails: while the final equations are the right ones, the method predicts some intermediate equations which are not generally satisfied, as can be readily seen in particular examples. Other methods, such as the method of the adjoint equations, also fail.

Therefore, one should be careful in using variational calculus. Instead of considering all the admissible variations we will find a certain class of variations, which are admissible and they are determined by complete lifts of sections.

Let $\sigma$ be a section of $\mathcal{A}$ such that $\sigma\left(m_{0}\right)=\sigma\left(m_{1}\right)=0$. We consider the vector fields $X=\rho(\sigma)$ and $Y=\rho^{1}\left(\sigma^{c}\right)$, and we denote by $\psi_{s}$ and $\Psi_{s}$ their respective flows. It follows that $\psi_{s}\left(m_{0}\right)=m_{0}$ and $\psi_{s}\left(m_{1}\right)=m_{1}$. The family of curves $\eta(s, t)=\Psi_{s}(\eta(t))$ is a 1-parameter family of admissible variations of $\eta_{0}$. Indeed, it is clear that the family $\eta(s, t)$ projects onto $\gamma(s, t)=\psi_{s}\left(\gamma_{0}(t)\right)$, and for every fixed $s$ we have that $\eta(s, t)$ is an 
admissible curve: on the one hand,

$$
\frac{\partial \gamma}{\partial t}(s, t)=T \psi_{s}\left(\dot{\gamma}_{0}\right)
$$

and on the other hand,

$$
\rho(\eta(s, t))=\rho\left(\Psi_{s}\left(\eta_{0}(t)\right)\right)=T \psi_{s}\left(\rho\left(\eta_{0}(t)\right)\right)=T \psi_{s}\left(\dot{\gamma}_{0}(t),\right.
$$

where we have taken into account that $\rho \circ \Psi_{s}=T \psi_{s} \circ \rho$. At the endpoints $t=t_{0}$ and $t=t_{1}$ we have

$$
\begin{aligned}
& \gamma\left(s, t_{0}\right)=\psi_{s}\left(\gamma_{0}\left(t_{0}\right)\right)=\psi_{s}\left(m_{0}\right)=m_{0}, \\
& \gamma\left(s, t_{1}\right)=\psi_{s}\left(\gamma_{0}\left(t_{1}\right)\right)=\psi_{s}\left(m_{1}\right)=m_{1} .
\end{aligned}
$$

The infinitesimal variation vector field $Z$ is $Z=Y \circ \eta_{0}$, and its projection is $W=X \circ \gamma_{0}$. Therefore, the variation of $L$ along $\eta(s, t)$ at $s=0$ is

$$
\frac{\partial(L \circ \eta)}{\partial s}(0, t)=Z(t) L=(Y L)\left(\eta_{0}(t)\right)=\left(\rho^{1}\left(\sigma^{c}\right) L\right)\left(\eta_{0}(t)\right)=d_{\sigma^{c}} L\left(\eta_{0}(t)\right),
$$

from where

$$
\left.\frac{d}{d s} J\left(\eta_{s}\right)\right|_{s=0}=\int_{\eta_{0}} d_{\sigma^{c}} L
$$

If $\sigma$ is a section satisfying the conditions given above, then so is $f \sigma$ for every function $f$ on $M$. Taking into account that $(f \sigma)^{c}=f \sigma^{c}+\dot{f} \sigma^{V}$, we have that

$$
\begin{aligned}
0 & =\int_{\gamma_{0}} d_{(f \sigma)^{c}} L \\
& =\int_{\gamma_{0}} \tilde{f} d_{\sigma^{c}} L+\dot{f} d_{\sigma^{V}} L \\
& =\left.\tilde{f}\left\langle\theta_{L}, \sigma^{c}\right\rangle\right|_{\eta_{0}\left(t_{0}\right)} ^{\eta_{0}\left(t_{1}\right)}+\int_{\eta_{0}} \tilde{f}\left\{d_{\sigma^{c}} L-d_{\Gamma}\left\langle\theta_{L}, \sigma^{c}\right\rangle\right\} \\
& =\int_{\eta_{0}} \tilde{f} i_{\sigma^{c}}\left\{d L-d_{\Gamma} \theta_{L}\right\},
\end{aligned}
$$

where we have taken into account that $\theta_{L}$ is semi-basic and $\sigma$ vanishes at $m_{0}$ and $m_{1}$. Since $f$ is arbitrary, we have that the vanishing of the variation of $J$ is equivalent to

$$
i_{\sigma^{c}}\left(d L-d_{\Gamma} \theta_{L}\right)=0,
$$

for every section $\sigma$ vanishing at $m_{0}$ and $m_{1}$. Since $\delta L=d_{\Gamma} \theta_{L}-d L$ is semi-basic and $\sigma$ is arbitrary we get the dynamical equations $\delta L=0$.

\section{References}

[1] J. F. Cariñena, J. Clemente-Gallardo, E. Follana, J. M. Gracia-Bondía, A. Rivero and J. C. Várilly, Connes' tangent groupoid and strict quantization, J. Geom. Phys. 32 (1999), 79-96.

[2] P. Libermann, Lie algebroids and mechanics, Arch. Math. (Brno) 32 (1996), 147-62.

[3] A. Weinstein, Lagrangian mechanics and groupoids, in: Mechanics Day, W. F. Shadwick et al. (eds.), Fields Institute Comm., Amer. Math. Soc., 1996. 
[4] E. Martínez, Lagrangian mechanics on Lie algebroids, Acta Appl. Math. 67 (2001), 295320 .

[5] J. Clemente-Gallardo, Applications of Lie algebroids in mechanics and control theory, in: Nonlinear Control in the New Millennium, F. Lamnabhi-Lagarrigue et al. (eds.), Springer, 2001, 299-313.

[6] A. Vaintrob, Lie algebroids and homological vector fields, Russian Math. Surveys 52 (1997), $428-29$.

[7] A. Vaintrob, Normal forms of homological vector fields, J. Math. Sci. 82 (1996), 3865-68.

[8] J. R. Camacaro and J. F. Cariñena, Batalin-Vilkovisky classical master equation and Lie algebroids, Publ. RSME 3 (2001), 215-220.

[9] R. Abraham and J. E. Marsden, Foundations of Mechanics, Benjamin, Reading, MA, 1978.

[10] G. Marmo, E. J. Saletan, A. Simoni and B. Vitale, Dynamical Systems: A Differential Geometric Approach to Symmetry and Reduction, Wiley, Chichester, 1985.

[11] M. Crampin, On the differential geometry of the Euler-Lagrange equations and the inverse problem of Lagrangian dynamics, J. Phys. A: Math. Gen. 14 (1981), 2567-2575.

[12] M. Crampin, Tangent bundle geometry for Lagrangian dynamics, J. Phys. A: Math. Gen. 16 (1983), 3755-3772.

[13] C. Chevalley and S. Eilenberg, Cohomology theory of Lie groups and Lie algebras, Trans. Amer. Math. Soc. 63 (1948), 85-124.

[14] J. F. Cariñena and L. A. Ibort, Noncanonical groups of transformations, anomalies, and cohomology, J. Math. Phys. 29 (1988), 541-45.

[15] I. Dorfman, Dirac Structures and Integrability of Nonlinear Evolution Equations, Wiley, Chichester, 1993.

[16] Y. Kosmann-Schwarzbach and F. Magri, Poisson-Nijenhuis structures, Ann. Inst. H. Poincaré Phys. Théor. 53 (1990), 35-81.

[17] B. Fuchssteiner, The Lie algebra structure of degenerate Hamiltonians and bi-Hamiltonian systems, Progr. Theor. Phys. 68 (1982), 1082-1104.

[18] G. Morandi, C. Ferrario, G. Lo Vecchio, G. Marmo and C. Rubano, The inverse problem in the calculus of variations and the geometry of the tangent bundle, Phys. Rep. 188 (1988), $147-284$.

[19] J. Grabowski and P. Urbański, Tangent and cotangent lifts and graded Lie algebras associated with Lie algebroids, Ann. Global Anal. Geom. 15 (1997), 447-486. 